\title{
Cratering and Cosmogenic Nuclides
}

Michael L. Blake and G. J. Wasserburg

The Lunatic Asylum of the Charles Arms Laboratory

Division of Geological and Planetary Sciences*

California Institute of Technology

Pasadena, California 91125

Abstract. A simple probabilistic model was constructed for the average value of a cosmogenic nuclide as a function of depth in a regolith. An arbitrary function was chosen for the size distribution of craters. The resulting integro-differential equation was found to reduce in limiting cases to: 1) the marching equation with a characteristic residence time, and 2) to the diffusion equation. The regolith diffusion constant is shown to be a simple integral of the cratering rate weighted by geometrical terms. This formal treatment provides a direct and general connection between cosmogenic nuclides and cratering rates and crater population in a simple analytical form. The validity of this model remains to be tested.

In this note we report on a simplified analysis of the relationship between cratering and mixing of a planetary surface and the production of nuclides produced by cosmic ray interactions with matter in the nuclear active zone near the surface. Let us consider the production rate of a specific nuclide to be only a function of depth and given by $\varphi(x)$. Take the origin to be at the surface. Let the average number of product nucleii per unit of volume at depth $\mathrm{x}$ and time $\tau$ be given by $\psi(\mathrm{x}, \tau)$. Then for a body with no mixing and no initial fuence it follows that $\psi(\mathrm{x}, \tau)=\tau \varphi(\mathrm{x})$ for a stable product nuclide. For a sampling area we consider an area $A_{O}$ which is far larger than any crater. Define $n(\xi) d \xi$ to be the probability that a crater of depth $\xi$ to $\xi+d \xi$ occurs in $A_{O}$ per unit time. We further assume for simplicity, that the geometry of a crater is of the form shown in Fig. 1. The thickness of a crater blanket from a crater of depth $\xi$ is taken as uniform and of value $h(\xi)$. We assume that the material in an ejecta blanket is well mixed. The total area of the blanket is taken to be $A(\xi)$ and the area of the cylindrical crater to be $a(\xi)$. If no material is lost from the planet during cratering, then we have the conservation equation $\mathrm{h}(\xi) \mathrm{A}(\xi)=\xi \mathrm{a}(\xi)$. Note that $\mathrm{h}(\xi) \rightarrow 0$ as $\xi \rightarrow 0$.

Now consider a surface at a time $\tau^{-}$(prior to an impact) with an average fluence or concentration as a function of depth given by $\psi\left(x, \tau^{-}\right)$. We inquire what the new average value of the product nuclide concentration is at a depth $x$ after a single cratering event to depth $\xi$. Subsequent to the cratering event at time $\tau^{+}$the areal average at depth $\mathrm{x}$ becomes

$$
\begin{aligned}
\psi\left(\mathrm{x}, \tau^{+}\right)= & {\left[\frac{\mathrm{A}_{\mathrm{O}}-\mathrm{A}(\xi)}{\mathrm{A}_{\mathrm{O}}}\right] \psi\left(\mathrm{x}, \tau^{-}\right)+\frac{\mathrm{A}(\xi)}{\mathrm{A}_{0} \xi} \int_{0}^{\xi} \psi\left(\eta, \tau^{-}\right) \mathrm{d} \eta\{\text { for } \mathrm{x}<\mathrm{h}(\xi)\} } \\
& +\left[\frac{\mathrm{A}(\xi)-\mathrm{a}(\xi)}{\mathrm{A}_{\mathrm{O}}}\right] \psi\left(\mathrm{x}-\mathrm{h}(\xi), \tau^{-}\right)\{\text {for } \mathrm{x}>\mathrm{h}\} \\
& +\frac{\mathrm{a}(\xi)}{\mathrm{A}_{0}} \psi\left(\mathrm{x}+\xi-\mathrm{h}(\xi), \tau^{-}\right)\{\text {for } \mathrm{x}>\mathrm{h}\}
\end{aligned}
$$

*Division Contribution Number 2657
The first term on the R-H-S corresponds to the undisturbed region exterior to regions I and II of Fig. 1c; if the depth $x<h$, then the only additional term is the second term corresponding to mixing; if the depth $\mathrm{x}>\mathrm{h}$ then the only additional terms are the last two corresponding to burial or excavation. Now we generalize this averaging procedure to include all classes of craters. Consider the craters produced in the time interval $\tau$ to $\tau+\delta \tau$, then we obtain

$$
\begin{aligned}
\psi(\mathrm{x}, \tau+\delta \tau)= & \left(1-\delta \tau \int_{0}^{\infty} \mathrm{n}(\xi) \frac{\mathrm{A}(\xi)}{\mathrm{A}_{0}} \mathrm{~d} \xi\right) \psi(\mathrm{x}, \tau) \\
& +\delta \tau \int_{\mathrm{h}^{-1}(\mathrm{x})}^{\infty} \mathrm{n}(\xi) \frac{\mathrm{A}(\xi)}{\mathrm{A}_{0}} \frac{\mathrm{d} \xi}{\xi} \int_{0}^{\xi} \psi(\eta, \tau) \mathrm{d} \eta \\
& +\delta \tau \int_{0}^{\mathrm{h}-1(\mathrm{x})} \mathrm{n}(\xi) \mathrm{d} \xi\left\{\frac{\mathrm{A}(\xi)-\mathrm{a}(\xi)}{\mathrm{A}_{0}} \psi(\mathrm{x}-\mathrm{h}(\xi), \tau)+\frac{\mathrm{a}(\xi)}{\mathrm{A}_{0}} \psi(\mathrm{x}+\xi-\mathrm{h}(\xi), \tau)\right\} \\
& +\delta \tau \varphi(\mathrm{x})-\delta \tau \lambda \psi(\mathrm{x}, \tau) .
\end{aligned}
$$

We have taken the decay constant of the product nuclide to be $\lambda$. The first term on the R-H-S is the probability that no crater occurs in time $\delta \tau$ multiplied by the value of $\psi$ at depth $\mathrm{x}$; the second term is the sum of the probabilities that cratering to depth $\xi$ takes place with blankets thicker than $x$ times the average value of $\psi$ in the blanket. The function $h^{-1}(x)$ is the inverse of the function $h(\xi)$ and is the depth of a crater whose blanket is of thickness $x$. The next two terms correspond to the probability of craters occurring with blankets thinner than $x$ times the value of $\psi$ as determined from the new surface; the last two terms are the production and decay of the product nuclide. Taking the limit as $\delta \tau \rightarrow 0$, we obtain the basic linear integro-differential equation:

$$
\begin{aligned}
\frac{\partial \psi(x, \tau)}{\partial \tau}= & \varphi(x)-\lambda \psi(x, \tau)+\int_{h^{-1}(x)}^{\infty} n(\xi) \frac{A(\xi)}{A_{0}}\left\{\frac{1}{\xi} \int_{0}^{\xi} \psi(\eta, \tau) d \eta-\psi(x, \tau)\right\} d \xi \\
& +\int_{0}^{h-1} n(\xi) d \xi\left(\frac{A(\xi)-a(\xi)}{A_{0}}\right)\{\psi(x-h(\xi), \tau)-\psi(x, \tau)\} \\
& +\int_{0}^{h-1} n(\xi) d \xi \frac{a(\xi)}{A_{0}}\{\psi(x+\xi-h(\xi), \tau)-\psi(x, \tau)\}
\end{aligned}
$$

Let the integrals on the R-H-S be called $I_{1}, I_{2}$ and $I_{3}$ as indicated. We define the function $\alpha(\xi) \equiv n(\xi) \frac{a(\xi)}{A_{0}}$. The function $\alpha(\xi) d \xi$ is the probability per unit area per unit time of the surface being excavated by a crater of depth $\xi$ to $\xi+\mathrm{d} \xi$. The moments of order i of the function $\alpha$ are given by

$$
\mu_{\mathrm{i}} \equiv \int_{0}^{\infty} \frac{\alpha(\xi) \xi^{\mathrm{i}} \mathrm{d} \xi .}{\mathrm{i} !}
$$


Note that from mass conservation $\mathrm{n}(\xi) \frac{\mathrm{A}(\xi)}{\mathrm{A}_{0}}=\frac{\alpha(\xi) \xi}{\mathrm{h}(\xi)}$.

From inspection of eqn. 3 it may be seen that the terms $I_{2}$ and $I_{3}$ vanish in the special case that $a(\xi)=A(\xi)$. This would correspond to a vertical mixing model in which all of the debris falls back into the crater. Models of this type have been discussed to varying degrees in the literature (see, for example, Curtis and Wasserburg, 1975) and an exhaustive analysis for this limiting case will be presented elsewhere (M. Blake, D. Curtis and G. J. Wasserburg).

Equation 3 can be simplified if we are far below the blanket depth, so that $h^{-1}(x) \rightarrow \infty$, and consider only deep craters such that the material beneath the crater bottoms is unirradiated. For this case there is no contribution from the vertical mixing term $I_{1}$, and $\psi(x+\xi-h, \tau)$ in term $I_{3}$ represents unirradiated material and is zero. Approximating $\psi(\mathrm{x}-\mathrm{h}, \tau)-\psi(\mathrm{x}, \tau) \approx-\mathrm{h} \frac{\partial \psi}{\partial \mathrm{x}}$ and neglecting terms of order $h / \xi$, we obtain

$\frac{\partial \psi(\mathrm{x}, \tau)}{\partial \tau}+\mu_{1} \frac{\partial \psi(\mathrm{x}, \tau)}{\partial \mathrm{x}}=-\left(\mu_{0}+\lambda\right) \psi(\mathrm{x}, \tau)+\varphi(\mathrm{x})$

For the case of a stable nuclide $(\lambda=0)$ the conservation of this species gives the boundary condition $\psi(0, \tau)=\frac{\mu_{0} \tau}{\mu_{1}} \int_{0}^{\infty} \varphi(\xi) \mathrm{d} \xi$ which may be written in the more general form:

$\psi(0, \tau)=\frac{\int_{0}^{\infty} \alpha(\xi) \mathrm{d} \xi \int_{0}^{\xi} \psi(\eta, \tau) \mathrm{d} \eta}{\mu_{1}}$

Equations 4 and 5 describe a system which is being buried by a rain of material deposited at a rate $\mu_{1}$ and which is subjected to mixing with a mean lifetime of $\frac{1}{\mu_{0}}$. The composition of the rain is given by (5) and corresponds to the average value of $\psi$ calculated over the volume of material excavated from different depths. For short times, before deep cratering mixes to a significant depth (so that $I_{3}$ may be neglected) and in the regime of depths much greater than crater blankets, the effect of deep cratering is to produce a continuous sedimentation blanket at a rate of $\mu_{1}$ and with a composition given by (5).

In general for larger values of time, such that $I_{3}$ may not be neglected, eqn. 3 holds. Study of this equation in conjunction with the conservation equation shows that $I_{2}+I_{3}$ may be written in the form:

$\int_{0}^{\mathrm{h}^{-1}} \alpha(\xi) \frac{\xi(\xi-\mathrm{h})}{2} \nabla_{\mathrm{XFD}}^{2} \psi(\mathrm{x}, \tau) \mathrm{d} \xi$

where $\nabla_{X F D}^{2}$ is the finite difference Laplacian at point $x$ corresponding to a non-uniform net at the points $x-h, x$, $\mathrm{x}+\xi-\mathrm{h}$.

$\nabla_{\mathrm{XFD}}^{2} \psi=\frac{\frac{\psi(\mathrm{x}+\xi-\mathrm{h}, \tau)-\psi(\mathrm{x}, \tau)}{\xi-\mathrm{h}}-\frac{\psi(\mathrm{x}, \tau)-\psi(\mathrm{x}-\mathrm{h}, \tau)}{\mathrm{h}}}{\left(\frac{\xi}{2}\right)}$

Approximating $\nabla_{\text {XFD with }}^{2} \nabla^{2}$ and neglecting terms of order $h / \xi$, we obtain the diffusion transport equation with the diffusion coefficient given by $\mu_{2}$ :

$\frac{\partial \psi(\mathrm{x}, \tau)}{\partial \tau}=\varphi(\mathrm{x})-\lambda \psi(\mathrm{x}, \tau)+\mu_{2} \nabla^{2} \psi(\mathrm{x}, \tau)$

$$
+\int_{\mathbf{h}^{-1}(\mathbf{x})}^{\infty} \mathrm{d} \xi \alpha \frac{\mathrm{A}(\xi)}{\mathrm{a}(\xi)}\left\{\frac{1}{\xi} \int_{0}^{\xi} \psi(\eta, \tau) \mathrm{d} \eta-\psi(\mathbf{x}, \tau)\right\}
$$

(a)

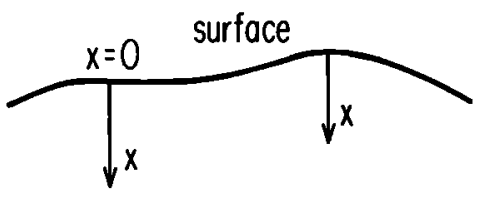

(b)

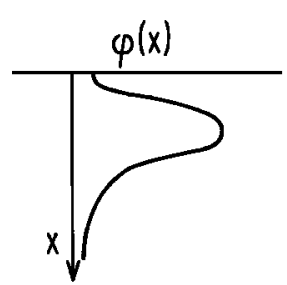

(c)

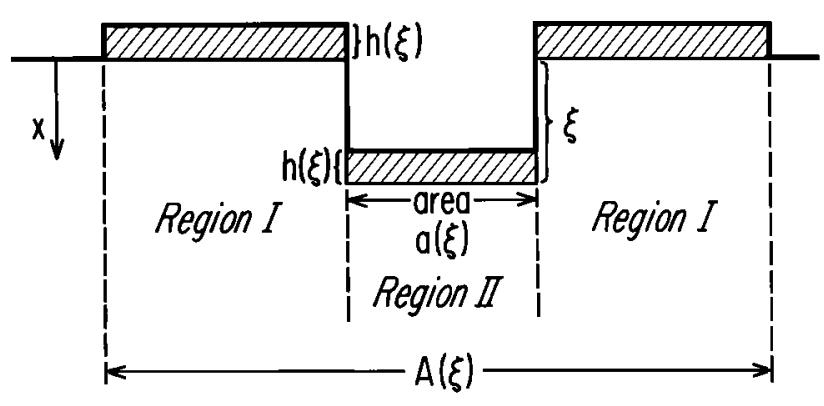

Fig. 1 (a) The boundary is located at $x=0$. Prior to an impact the fluence at depth $\mathrm{x}$ is represented by the areal average $\psi(x, \tau)$ at that depth. (b) The production of a nuclide is given by $\varphi(x)$. (c) After a cratering event of depth $\xi$, the well-mixed blanket of thickness $h(\xi)$ shown by the hatchured area is assumed to cover an area $A(\xi)$. In region I for $x>h(\xi)$ the fluence at depth $\mathrm{x}$ is given by $\psi(\mathrm{x}-\mathrm{h}, \tau)$ and in region II is given by $\psi(\xi-h+x, \tau)$. In the undisturbed region exterior to I and II, the fluence is given by $\psi(\mathrm{x}, \tau)$. For $\mathrm{x}<\mathrm{h}$ in regions I and II the fluence is given by the average $\frac{1}{\xi} \int_{0}^{\xi} \psi(\eta, \tau) \mathrm{d} \eta$.

For thin blankets $(h \rightarrow 0)$ we may again ignore the vertical mixing term and obtain the simple diffusion equation with a source-

$\frac{\partial \psi(\mathrm{x}, \tau)}{\partial \tau}=\varphi(\mathrm{x})-\lambda \psi(\mathrm{x}, \tau)+\mu_{2} \nabla^{2} \psi(\mathrm{x}, \tau)$

Assuming $\psi$ and its derivatives are smooth and vanish at infinity, we obtain for the boundary condition

$\left(\frac{\partial \psi(x, \tau)}{\partial x}\right)_{x=0}=0$

Using eqn. 3 the boundary condition in the limit $h \rightarrow 0$ may also be shown to be

$\psi(0, \tau)=\frac{\int_{0}^{\infty} \alpha(\xi) \frac{\xi}{h(\xi)}\left\{\frac{1}{\xi} \int_{0}^{\xi} \psi(\eta, \tau) \mathrm{d} \eta\right\}}{\int_{0}^{\infty} \alpha(\xi) \frac{\xi}{\mathrm{h}(\xi)} \mathrm{d} \xi}$

Note that this is an areal average and not a volume average as obtained for the rain equation.

It follows that in this regime the governing transport equation is the diffusion equation with a source, with the diffusion coefficient given by $\mu_{2}$. This result permits the easy use of a wide variety of well known analytical and numerical solutions. The diffusion type behavior is independent of the particular cratering law and only depends on the second moment; however, the onset of diffusion behavior is governed by the time constant $\tau \sim \mathrm{x} / \mu_{1}$. In general, the behavior will lie between the marching behavior (eqn. 4 ) and the diffusive regime (eqn. 7).

We conclude that this simple geometrical model provides a 
physically consistent description of the relationship between the concentration of nuclides and the cratering rates. The key parameters are the first three moments of the cratering law $\left(\mu_{0}, \mu_{1}, \mu_{2}\right)$. The possibility of a diffusion approximation for lateral regolith transport is referred to by Shoemaker inter multa alia (1970). We believe that the formal analysis presented here may possibly be extended to the lateral problem as well.

The functional form of the diffusion coefficient $\mu_{2}$ is of some interest as it indicates the extent to which the average transport properties depend upon crater size and frequency. We note that since $a(\xi)$ goes like $\xi^{2}$ then the integrand in the expression defining $\mu_{2}$ goes like $n(\xi) \xi^{4}$. Hence, neglecting details of crater geometry, it follows that the condition that craters of different sizes make equal contributions to transport is $\mathrm{n}(\xi) \propto \xi^{-4}$. The power laws found from crater counts [see Shoemaker inter multa alia (1970)] are close to this limit and shows that a large fraction of the transport must be due to big craters.

For the diffusive limit (eqn. 7), the solution at $x=0$ for the source $\varphi(x)=\varphi_{0} \delta\left(x-x_{0}\right)$ is, to second order,

$$
\psi(0, \tau) \cong \frac{\varphi_{0} \tau}{\left(\pi \mu_{2} \tau / 4\right)^{1 / 2}}\left\{1-\frac{\mathrm{x}_{0}}{\left(4 \mu_{2} \tau / \pi\right)^{1 / 2}}\right\}
$$

If we assume for the purposes of calculation that the lunar regolith is in the diffusive regime, then we may calculate the diffusion coefficient from the neutron capture data on $\mathrm{Gd}$. Using the values $\varphi_{0}=1.7 \times 10^{19} \mathrm{n} \mathrm{gm} /\left(\mathrm{cm}^{4} \mathrm{AE}\right), \quad \psi(0,3.8 \mathrm{AE})$ $=2.3 \times 10^{16} \mathrm{n} / \mathrm{cm}^{2}, \quad \tau=3.8 \mathrm{AE}, \quad \mathrm{x}_{0}=1$ meter and assuming $\mathrm{a}$ density of $2 \mathrm{~g} / \mathrm{cm}^{3}$, we obtain $\mu_{2}=58$ meters $^{2} / \mathrm{AE}$. This result should be self consistent with that obtained for other nuclides for which the total production rate is known.

The relationship between depth and time scale is approximately given by $x / \sqrt{\mu_{2} \tau} \sim 1 / 2$ for $50 \%$ survival. This is roughly compatible with the existing data on lunar cosmogenic nuclides for the value of $\mu_{2}$ as given and may possibly be compatible with regolith depths. It yields a far more rapid turnover time than suggested by Gault, Hörz, Brownlee and Hartung (1974) using the flux of primary bombarding objects.

It is most satisfying that so complex a problem appears amenable to analytical treatment. The major inadequacy of this model is the neglect of downslope transport and the treatment of a discrete problem as a continuous problem. The simplified crater geometry that was used does not seem to us to be a severe limitation. A more complete report on the comparison of analytical and numerical results and a comparison with different cratering laws and crater blanket characteristics will be reported more fully later.

This work was supported by grants from the National Aeronautics and Space Administration (NGL 05-002-188) and the National Science Foundation (MPS-7102670 A05). One of us would like to thank T. Tombrello for pointing us West during Michael Blake's senior year. We are pleased to acknowledge fervent discussions, continuous interaction and aid from our colleague David B. Curtis.

\section{References}

Curtis, D. B. and G. J. Wasserburg, Apollo 17 Neutron Stratigraphy, in press, The Moon (1975).

Gault, D. E., F. Hörz, D. E. Brownlee, and J. B. Hartung, Mixing of the lunar regolith, Geochim. Cosmochim. Acta, Supplement 5 Vol. 3 p. 2365, 1974.

Shoemaker, E. M., M. H. Hait, G. A. Swann, D. L. Schleicher, G. G. Schaber, R. L. Sutton, D. H. Dahlem, E. N. Goddard, A. C. Waters, etc. Origin of the lunar regolith at Tranquility Base, Geochim. Cosmochim. Acta, Supplement 1, Vol. 3, p. 2399, 1970.

(Received August 14, 1975; accepted September 11, 1975.) 